\title{
Standards for Ethics in Sport and Exercise Science Research: 2018 Update
}

\author{
Authors \\ D. J. Harriss', A. Macsween², G. Atkinson²
}

\section{Affiliations}

1 Research Institute for Sport and Exercise Sciences, Liverpool John Moores University, Liverpool, UK

2 Health and Social Care Institute, School of Health and Social Care, Teesside University, Middlesbrough, UK

Bibliography

DOI https://doi.org/10.1055/s-0043-124001

Int J Sports Med 2017; 38: 1126-1131

(C) Georg Thieme Verlag KG Stuttgart · New York

ISSN 0172-4622

\author{
Correspondence \\ D. J. Harriss \\ Research Institute for Sport and Exercise Sciences \\ Tom Reilly Buidling \\ Byrom Street Campus \\ Liverpool John Moores University \\ Webster Street \\ Liverpool L3 3AF \\ United Kingdom \\ Tel.: +44/15/19 0462 36, Fax: +44/15/12 073224 \\ D.Harriss@ljmu.ac.uk
}

For publication in the International Journal of Sports Medicine (IJSM), studies involving human participants or animals must have been conducted in accordance with recognised ethical standards and national/international laws. At the very first stage of paper submission, authors are required to adhere to these and all other relevant standards and laws.

In the $21^{\text {st }}$ Century, research opportunities, approaches and environments are in a continual state of flux, and this is also the case for the associated ethical issues. In the original 2009 IJSM editorial [2], we described the ethical considerations embedded into national/international laws and provided specific guidance on the ethical issues which commonly arise in Sports Medicine research. In 2011 , this information was updated to recognise the ethical principles of other professional associations and treaties when conducting research involving human participants [3]. Additional information was also provided on the use of Laboratory Animals in research, and on the links between sample size and research ethics. In the second update, published in 2013, we elaborated on the ethical issues relating to the investigation of doping agents; the use of animals for answering research questions that appear to be solely focussed on the enhancement of athletic performance; and sample size in the context of the burden to individual research participants [4]. In 2015, we updated some of the guidelines to account for the changes made to the Declaration of Helsinki in 2013, covered the use of social media in research, provided guidance on how researchers can feed back their incidental and pertinent findings to research participants, covered some of the issues relating to studies involving children, and outlined the difference between a full and pilot study in terms of desired number of participants [5].
In this, our new update for 2018 onwards, we provide the following revisions and additions, labelled with the word "Update" in bold text at various points in this paper;

- Clarification of the issues surrounding the use of a gatekeeper for accessing personal information on participants.

- Clarification of some of the requirements for research with participants who are either too young to give a valid consent (under relevant local Statute) themselves and/or who lack the mental capacity to give an informed consent.

- Clarification of the expectations for the presentation and content of information given to facilitate informed consent/ assent.

- Highlighting of the obligation to breach confidentiality in certain circumstances and the importance of communicating all relevant process to the potential participant.

- Clarification on the use of person identifiable information including a reminder of the upcoming (May 2018) changes to legislation governing how personal data may be accessed and processed in research in the European Union (https://www. eugdpr.org/).

- Provision of advice surrounding the increasing calls for open access data, including the resulting data storage issues.

- Update on the ethical and legal considerations involved in secondary analysis (retrospective data) studies.

- Clarification on the use of placebos in research.

- Coverage of the expectations for studies that involve participant deception (i. e. where fully informed consent is not obtained in advance of participation).

- Elaboration of the sample size issues relevant to research ethics. 
- Coverage of the issues surrounding research on contracted athletes, who are obliged to have data collected on them as part of their contract.

- Highlighting of the issues surrounding participant withdrawal from a study

- Update on the importance of study registration on a public database and the publication of study protocols

- Update on sample size issues, including the recent concerns about reproducibility of study results

- Clarification of expectations for the reporting of adverse events/reactions.

\section{Research Involving Human Participants}

Authors should conduct their research involving human participants ethically according to the principles of the Declaration of Helsinki [21]. The Declaration is intended to be read as a whole and every principle is equally important, but those points most-commonly considered by sport and exercise scientists are summarised below.

1. Basic principles. the protection of participant's dignity, safety, rights and wellbeing take precedence over all other interests.

2. Ethical review. Before research begins and before any amendments are made after the research begins, the research must be reviewed and approved by appropriate ethics committee/ institutional review board.

3. The research protocol. The study, research design and statistical analysis must be clearly described, justifiable and appropriate. In drawing up the research protocol, the researcher must;

a. Consider ethical issues in accordance with the Declaration of Helsinki,

b. Provide information regarding funding, insurance, sponsorship, institutional affiliations, and any other potential conflicts of interest,

c. Consider the contribution to new knowledge and the environment,

d. Ensure that considerations have been given to compensation and treatment arrangements should any participants be harmed as a result of their taking part.

e. Consider the arrangements for post-study access, by all participants, to interventions identified as beneficial in the study or access to other appropriate care or benefits.

4. Update: Gatekeepers - A gatekeeper is any person, or organisation, that acts as an intermediary between a researcher and potential participants (e.g., health care professionals, administrators or managers of Schools, sports clubs, treatment service providers etc. or coaches, instructors etc.). NB - Where a gatekeeper identifies and contacts potential participants, on behalf of a researcher, those contacted who are interested in taking part should normally be directed (in the Participant Information Sheet) to contact the researcher directly themselves and not the gatekeeper, to ensure their choice is not known to the gatekeeper. The use of a gatekeeper is necessary:

a. Where the researcher does not have a pre-existing, legitimate, legal basis (legal under relevant Data Protection Legislation) to access, or process, personal information that is required to screen for eligibility and/or identify and contact potential participants or. b. Where the researcher needs to access or use premises (such as facilities, or staff) to undertake their research which they do not have a pre-existing, legitimate right to access or use.

5. Update: Consent. Informed consent/assent should normally be provided freely and in confidence, by the participant and should ideally be recorded in writing. If written consent/assent cannot be obtained, or is not appropriate, then oral consent/assent should be formally documented and witnessed. The information given for consent/assent must be given in an appropriate format and language for the target population. Asking people who would be eligible for the study, to review study materials (particularly consent/ assent and recruitment materials) - in their capacity as expert consultees - before commencing the research is highly recommended [13]. Information given must include (as a minimum) the;

a. names and affiliations of those undertaking the research (in particular the Chief Investigator)

b. aims of the research

c. what will be required of those who take part

d. sources of funding

e. conflicts of interest

f. the name of the study sponsor

g. anticipated benefits and potential risks

h. potential discomfort

i. right to refuse to participate or withdraw consent without reprisal

j. payments of expenses and/or incentives to take part

k. details of who to contact to ask questions about the study

I. the name of the Research Ethics committee/Institutional Review Board (REC/IRB) that approved the study.

6. Update: Consent from young people. In research projects that recruit people who are too young to give a valid consent (under relevant local Statute) themselves, consent should be obtained, from an appropriate person (parent/guardian), that the young person may be invited and may take part if they wish to; assent should then be obtained from the participant. Only where consent and assent are obtained should a person who is too young to give a valid consent (under relevant local Statute) themselves normally be eligible.

7. Update: Capacity for consent. Research projects that recruit people who lack the mental capacity to give an informed consent (for any reason) may be undertaken only if the study aims could not be achieved by recruiting from a population who were able to give consent. Studies involving participants who lack the Mental Capacity to give an Informed Consent may only be conducted (in the UK) if Health Research Authority Approval has been confirmed (corresponding international standards must also be adhered to).

8. Update: Conduct of Research.

a. in accordance with appropriate consideration of risk

b. by appropriately qualified researchers and support staff

c. with skill and care

d. in an appropriate setting

e. in a way that protects the privacy of participants and confidentiality of their personal information

f. in accordance with laws and regulations of the country or countries in which the research is to be performed as well as international norms and standards. Specific laws relevant 
to research ethics may regulate, for example: the collection, use and/or storage of human tissue; the protection of individuals that lack the capacity to consent; data protection; and the use of drugs in research etc..

9. Update: Governance. Adverse events/reactions, occurring during the study, must be reported to the Sponsor's representative and the relevant REC/IRB Chair as soon as possible.

10.Update: Limits of confidentiality - Researchers should be aware of the circumstances when participant confidentiality should be breached. For example; in the UK, researchers are legally-obliged to breach confidentiality if they observe or learn about physical or sexual abuse of children, and crimes that contravene prevention of terrorism legislation. Researchers may also have a professional obligation to breach confidentiality. For example; a registered sports nutritionist might be obliged to report anything they observe or learn about the use of drugs in sport. Participants must be fully informed (in the participant information sheet) of the limits of confidentiality and the process and actions if confidentiality needs to be breached.

\section{Researcher Obligations}

Authors may conduct their research in accordance with principles detailed by professional associations and treaties other than the World Medical Association Declaration of Helsinki such as the International Sociological Association's (ISA) Code of Ethics [12]. This update recognises that differences in ethical principles may exist between professional associations. For example, the ISA's code of Ethics states that "The consent of research subjects and informants should be obtained in advance. Covert research should be avoided in principle, unless it is the only method by which information can be gathered, and/or when access to the usual sources of information is obstructed by those in power." [12]. Authors are required to confirm whether aspects of their research abide by ethical principles set down by professional associations or treaties that differ in status to the Declaration of Helsinki.

Author(s) should be able to confirm that the following points are upheld [8]:

1. That consent to participation was robust, such that the participants were provided with adequate information, the consent was given voluntarily and that those providing consent were competent to do so - or - that an alternative consent process was approved by the relevant REC/IRB.

2. If research was carried out with participants who were vulnerable or unable to provide consent, then the authors confirm that the participants were appropriately identified, approached and recruited into the study, there was justification for carrying out the research on these individuals and additional measures were put in place to ensure the research was ethical.

3. Issues of privacy and confidentiality have been considered beyond what is legally required. Where, "privacy is the protection of control over information about oneself; control over access to oneself, both physically and mentally; and control over one's ability to make important decisions about family and lifestyle in order to be self-expressive and to develop varied relationships" [8] and confidentiality is when the "participant dis- closes to the researcher information which the participant regards as confidential or secret [default assumption]; and the researcher undertakes (implicitly or explicitly) not to reveal this information to anyone who does not already possess it." [8].

4. Researchers have considered their legal and ethical obligations if privacy and confidentiality are breached.

5. Update: Person identifiable information. If person identifiable information, provided as part of a research study is to be accessed or processed, for purposes other than those covered by the original informed consent the participant's consent for that should normally be obtained and documented - or - the legal basis for that access and processing must be detailed and the justification for not obtaining explicit consent must be persuasive.

6. Risks relating to harm, inconvenience, time and money, as well as any benefits to the participant, to other individuals, to the researchers and organisations have been considered in a balanced fashion, communicated to the participants and appropriately managed.

7. Participants have not been exploited and that particular groups have not been discriminated from participating in the research.

8. There are appropriate governance arrangements and structures in place if participants are asked to donate biological material for use in future research, such as a "biobank". These arrangements should involve appropriate consideration of broad consent, privacy and confidentiality, feedback to the participant of incidental findings, storage of material, commercial involvement, donor involvement and intellectual property rights and must comply with local statute.

9. Update. Open access data. Many funding bodies and journal editors now ask for data collected as part of a study to be made available to the scientific community and the public. We advise that all researchers now consider the likelihood of future secondary data analysis and data sharing.

\section{Research Involving Animals}

Authors should conduct their research involving animals ethically according to the principles of the Guide for the Care and Use of Laboratory Animals of the Institute for Laboratory Animal Research [9]. Again, the guide is intended to be read as a whole, but the basic obligations on the researcher are summarised below. The researcher must;

a. Ensure the appropriateness of experimental methods

b. Legally acquire animals

c. Ensure that animals are properly housed and fed to ensure safe, hygienic and comfortable living conditions.

d. Maintain a record of animal care

e. Ensure that animal maintenance and research are carried out by qualified personnel, following all legal statutes and regulations

f. Administer appropriate pain management to minimize suffering, discomfort and pain

The eighth edition of the Guide for the Care and Use of Laboratory Animals [10], published in 2010, includes expanded coverage of the ethics of laboratory animal use; components of effective Animal Care and Use Programs; and new guidelines for the housing, 
environment, and enrichment of terrestrial and aquatic animals, and for veterinary and clinical care [11]. Specifically:

a. The core foundation of the guide - replacement, refinement, and reduction.

b. An "Animal Care and Use" Program

c. The performance standards approach for animal care and care practices

d. The care and use of fish and other aquatic species

e. Housing space and enclosures for animals' social needs

$\mathrm{f}$. Environmental enrichment got the enhancement of animal well-being to provide sensory and motor stimulation and promote psychological health

g. Discussion of animal biosecurity practices

\section{Exercise protocols in animal research}

A useful document for any researcher interested in studying animals in an exercise context is the Resource Book of the American Physiological Society [15]. It is clear that the study of animals can help elucidate the mechanisms of exercise-related benefits to both human and animal health. Nevertheless, any animal study that has been specifically designed to answer a research question based solely on the enhancement of human athletic performance should include a clear explanation as to why such a study is necessary, and why it could not be undertaken on humans. This rationalisation is important not just from an ethical perspective but is in keeping with the aim of maximising external validity in any study.

\section{Other Important Issues}

1. Update: Secondary data analysis. Data are routinely collected from individuals, for various purposes; e.g. sport scientists may monitor and record, data on physiological function of an athlete in order for him or her to gain an edge over their rivals. Person identifiable data collected for one purpose (e.g. monitoring of performance, or training) cannot be accessed, or processed, for another purpose (e.g. research), unless explicit consent for that other use is obtained, that other use is ethically approved and relevant data protection legislation is complied with. Ethical approval granted for a research study, which has already begun is invalid.

2. Update: The use of placebo. Ideally, participants should be randomly assigned, with adequate concealment approaches, to experimental or placebo arms. Consideration should always be given to:

a. Allowing participants in both arms access to the intervention after a defined period of testing (e.g. cross-over designs). In cross-over designs the sequence of study conditions is usually randomised for each participant,

b. Arrangements for monitoring (e.g. including interim analysis of un-blinded data) to determine if people in either arm are fairing more poorly [18], thus enabling independent judgements as to whether the study should be halted (e.g. establishing an independent data monitoring and ethics committee).

c. In research where participants are patients, or vulnerable for some other reason, particular care must be taken. The use of placebo is generally considered ethical only where there is both genuine equipoise as to the efficacy/effective- ness of the intervention under study, in the population under study - and - when the participants will not be at undue, or disproportionate, risk.

3. Update: Deception. The use of deception in research (e.g. in a pacing strategy study in which time trial distance is deceived) is normally only acceptable where the study aims could not be achieved if informed consent were obtained - and - the likely benefits of the study (e.g. benefits from dissemination of findings) outweigh the compromising of the principle of informed consent. Where there is any risk of e.g. pain or emotional distress, from participation, deception is expected to only extend to deception about the purpose/aims of the study, not, about what participants will be asked to do and the risks; i.e. participants should normally be accurately informed of the processes they will be asked to undertake and the risks, before consenting even where the purpose of the study and or outcomes of interest, are not revealed until after participation Participants must be debriefed and fully informed, at the conclusion of the study and then given the option to withdraw their data [19].

4. Good research practice. The UK Medical Research Council has outlined some basic principles of good research practice which if followed will help to ensure that research is conducted ethically. These include planning, conduct, recording data, reporting results, applying the results [16]. Relevant issues not specifically raised in the Declaration of Helsinki include the use, calibration and maintenance of equipment, Control of Substances Hazardous to Health, documentation of standard operating procedures, retention of data, publication policy, authorship, correction of errors and retraction of published findings and intellectual property rights.

5. Athletes as participants in studies on doping agents.In principle, recreational and elite athletes should not be recruited to participate in research that exposes them to violations of the World Anti-Doping Code. However, there may be value of research into doping in sport - and so a desire to recruit athletes as participants. Investigators who wish to recruit athletes as participants in research involving performance enhancing substances and methods should consider the following:

a. Consultation with appropriate and relevant authorities (specific to each individual athlete) such as research ethics committees, WADA, international sport federations and national anti-doping organisations - to help protect recreational athletes, elite athletes and sport.

b. An unfair advantage should not be afforded to a recreational or elite athlete participating in the research.

c. "Adequate precautions should be taken so that the results of research are not misused and applied for doping" [20]

d. Append the WADA letter entitled "Scientific research using elite athletes: WADA point of view" [7] to the participant information sheet to help fully inform participants who are recreational or elite athletes.

6. Ethics and sample size. Statistical power should be considered by all authors submitting to IJSM. Ideally an a priori estimation of the minimal sample size for adequate statistical power and/ or adequate precision of a confidence interval should be reported. Authors and reviewers of IJSM manuscripts should be aware of the following important points: 
a. The minimal sample size for adequate statistical power should be considered alongside the burden of the study procedures on individual participants/animals and the predicted importance of the study findings to the knowledge base as well as to the impact on real-world practice [1]. An unethical scenario is where participants, or animals, have been burdened by the study procedures, but the study is likely to generate findings that have dubious clinical/practical importance. A "small" study might not be unethical, especially if participant burden is low and clinical/practical importance of the study findings are high, even if "statistical significance" has not been realised.

b. For the importance of a study to be judged, it is imperative that the minimal clinically/practically important magnitude of change or difference (MCID) is justified clearly and reported by authors [6]. We encourage authors to report the associated confidence interval(s), at least for the primary study outcomes. Authors who rely solely on statistical significance to judge clinical/practical importance will have their manuscripts rejected.

c. Update: False findings in research. It is wholly inappropriate for a reviewer to criticise a study on the basis of a perceived small sample size without considering the above issues of participant burden and clinical/practical importance. Nevertheless, researchers should be aware of current discussions concerning the so-called "replication crisis" in research. John loannidis has described the main issues in this context [14]. In brief, a study finding is less likely to be true when the sample sizes and effect sizes are small; when there is more "data mining", when there is less adherence to formal research frameworks such as CONSORT, and when more research groups in a topic area are interested in finding statistical significance. The ethical issue is, again, whether human participants or animals should be encouraged to volunteer for a particular study that may be more likely to lead to a false finding.

d. There are useful guidelines on what constitutes a pilot or feasibility study, e.g. those from the National Institute for Health Research: http://www.nihr.ac.uk/CCF/RfPB/FAQs/ Feasibility_and_pilot_studies.pdf. It is worth bearing in mind that these studies might not necessarily need to be powered to detect a certain effect size. Alternatively, they may be powered to detect, with adequate precision, a standard deviation for use in estimations of the required sample size for a future adequately powered trial of efficacy/effectivenes.

7. All research which involves the use of social media to recruit participants, conduct research or as a data source must:

a. be ethically reviewed and approved by an appropriate REC/ IRB

b. ensure that the principles of informed consent; risks, burdens and benefits; and privacy and confidentiality are adhered to. Researchers should address the following issues; the practicalities of providing information to ensure informed consent can be documented and collecting/using data; anonymity, privacy and confidentiality, in a setting where information is publicly available, from identifiable sources; the potential for harm and intrusion; data owner-

ship and security; researcher identity.

8. Pertinent findings are related to the variable or primary outcome(s) being studied and incidental findings are not related to the variable or primary outcome(s) being studied. Both pertinent and incidental findings may be interesting, or important to research participants and it is the responsibility of researchers to decide whether to feedback findings, provide clear information to participants, seek consent, respect autonomy and understand and manage expectations by having a practical feedback pathway that is adequately resourced.

9. In accordance with recommendations made by the Nuffield Council on Bioethics to the Health Research Authority (HRA) [17], researchers should endeavour to involve children, young people and parents/guardians, as appropriate, in the design of research. Participant involvement in other aspects of the research such as the dissemination of findings is also good practice [13].

\section{Conflict of Interest}

The authors declare no conflict of interest.

\section{References}

[1] Bacchetti P, Wolf LE, Segal MR, McCulloch CE. Ethics and sample size. Am J Epidemiol 2005; 161: 105-110

[2] Harriss D], Atkinson G. International Journal of Sports Medicine ethical standards in sport and exercise science research. Int J Sports Med 2009; 30: 701-702

[3] Harriss D], Atkinson G. Update - Ethical Standards in Sport and Exercise Science Research. Int J Sports Med 2011; 32: 819-821

[4] Harriss D], Atkinson G. Ethical standards in sport and exercise science research: 2014 update. Int J Sports Med 2013; 34: 1025-1028

[5] Harriss D], Atkinson G. Ethical standards in sport and exercise science research: 2016 update. Int J Sports Med 2015; 36: 1121-1124

[6] Hopkins WG, Marshall SW, Batterham AM, Hanin J. Progressive statistics for studies in sports medicine and exercise science. Med Sci Sports Exerc 2009; 41: 3-12

[7] Howman D. Scientific research using elite athletes: WADA point of view. J Appl Physiol 2013; 114: 1365-1365

[8] Hughes J, Hunter D, Sheehan M, Wilkinson SD, Wrigley A, Hughes J. European textbook on ethics in research. Luxembourg: Publications Office of the European Union; 2010: 203

[9] Institute for Laboratory Animal Research, National Research Council. Guide for the Care and Use of Laboratory Animals. Washington, D.C: National Academy Press; 1996: 140

[10] Institute for Laboratory Animal Research, National Research Council. Guide for the Care and Use of Laboratory Animals. Washington, D.C: National Academy Press; 2010: 248

[11] Institute for Laboratory Animal Research, National Research Council. Guide for the care and use of laboratory animals - report in brief. Washington, D.C.: National Academy Press; 2010 Available at http:// dels.nas.edu/resources/static-assets/materials-based-on-reports/ special-products/guide_lab_animals_final.pdf 
[12] International Sociological Association. Code of Ethics.Madrid: International Sociological Association; 2001 Available at http://www. isa-sociology.org/about/isa_code_of_ethics.htm

[13] Involve and HRA: New Health Research Authority (HRA) and INVOLVE briefing and guidance on public involvement and ethical review; Available at http://www.invo.org.uk/posttypenews/new-healthresearch-authority-hra-and-involve-briefing-and-guidance-on-publicinvolvement-and-ethical-review/?style = print

[14] loannidis JPA. Why most published research findings are false. PLoS Med 2005; 2: e124

[15] Kregel KC. Resource book for the design of animal exercise protocols. Committee to develop an American physiological society resource book for the design of animal exercise protocols 2006 Available via http://www.the-aps.org/pa/action/exercise/book.pdf Accessed 24 October 2013

[16] Medical Research Council. MRC ethics series: Good research practice 2005. London: Medical Research Council; 2005: 1-16
[17] Nuffield Council on Bioethics: Children and Clinical Research: Ethical Issues. . HRA Response to Report Recommendations Relevant to the Health Research Authority (HRA) and Research Ethics Committees (RECs). Available at http://www.hra.nhs.uk/documents/2015/08/ hra-response-ncob-children-clinical-research-recommendations-hrarecs-v1-0-final.pdf

[18] Shephard R]. Ethics in exercise science research. Sports Med 2002; 32: 169-183

[19] The American Psychological Association. Ethical principles of psychologists and code of conduct. Am Psychol 2002; 57: 1060-1073

[20] World Anti Doping Code. World Anti Doping Agency. Available at http://stage.wada-ama.org/Documents/World_Anti-Doping_Program/ WADP-The-Code/WADA_Anti-Doping_CODE_2009_EN.pdf

[21] World Medical Association. Declaration of Helsinki: Ethical principles for medical research involving human subjects. JAMA 2013; 310: 2191-2194 